hep-th/0406057

\title{
Thermodynamics and Stability of Hyperbolic Charged Black Holes
}

\author{
Rong-Gen Cai* \\ CASPER, Department of Physics, Baylor University, Waco, TX76798-7316, USA \\ Institute of Theoretical Physics, Chinese Academy of Sciences, P.O. Box 2735, Beijing 100080, China \\ Anzhong Wang ${ }^{\dagger}$ \\ CASPER, Department of Physics, Baylor University, Waco, TX76798-7316, USA
}

\begin{abstract}
In AdS space the black hole horizon can be a hypersurface with a positive, zero or negative constant curvature, resulting in different horizon topology. Thermodynamics and stability of black holes in AdS spaces are quite different for different horizon curvatures. In this paper we study thermodynamics and stability of hyperbolic charged black holes with negative constant curvature horizon in the grand canonical ensemble and canonical ensemble, respectively. They include hyperbolic Reissner-Nordström black holes in arbitrary dimensions and hyperbolic black holes in the $\mathrm{D}=5,4,7$ gauged supergravities. It is found that the associated Gibbs free energies are always negative, which implies that these black hole solutions are globally stable and black hole phase is dominant in the grand canonical ensemble, but there is a region in the phase space where black hole is not locally thermodynamical stable with a negative heat capacity for a given gauge potential. In the canonical ensemble, the Helmholtz free energies are not always negative and heat capacities with fixed electric charge are not always positive, which indicates that the Hawking-Page phase transition may happen and black holes are not always locally thermodynamical stable.
\end{abstract}

\section{INTRODUCTION}

Black holes in anti-de Sitter (AdS) spaces are quite different from black holes in flat or de Sitter (dS) spaces. In asymptotically flat or dS spaces, the horizon topology of a four dimensional black hole must be a round sphere $S^{2}$ [1]. In AdS spaces, except for the positive constant curvature horizon, it is possible to have black holes with zero or negative constant curvature horizons [2]-[8]. Due to the different horizon structure, the associated thermodynamic properties of black holes are rather different. Indeed, for the Schwarzschild black holes in AdS spaces, there is a phase transition (named as Hawking-Page phase transition) between the high temperature black hole phase and low temperature thermal AdS space 9]. In the AdS/CFT (conformal field theory) correspondence [10], thermodynamics and phase structure of black holes in AdS spaces can be mapped to those of dual CFTs. It has been argued by Witten [1] that the Hawking-Page phase transition of Schwarzschild black holes in AdS spaces can be identified with confinment/unconfirment phase transition of dual CFTs. As for the phase transition, it has been indeed found that for the AdS black holes with zero or negative constant curvature horizon, the Hawking-Page phase transition does not appear and black hole are always locally thermodynamically stable with positive heat capacity [4] (see also $[2,3,[5,6])$.

The electric charge of AdS black holes can be mapped to the R-charge of supersymmetric dual CFTs 12, 13, 14, [15, 16]. Therefore it is of equal interest to study thermodynamics and phase structure of AdS charged black holes. Indeed, it has been shown that phase structure of AdS charged black holes is rather rich because of the presence of charge, for example, see [3, 5, 6, 14]. However, most studies on the AdS charged black holes are restricted to the case with black hole horizon being a positive constant curvature hypersurface. Note that the fact that AdS black holes with zero curvature horizon can be the large horizon limits of the AdS black holes with positive constant curvature horizon [6, 11, 14], and that the so-called hyperbolic AdS black holes, namely, AdS black holes with negative constant curvature horizon, belong to another branch. It is therefore of necessary to investigate thermodynamics and phase structure of hyperbolic charged black holes and to see what main differences are among AdS charged black holes with different characteristic curvature horizons. This is just the goal of the present paper.

When the gauge charge is present, we can consider two different ensembles to study thermodynamics of charged black holes [17]. One is the grand canonical ensemble in which the gauge potential (chemical potential conjugate to the electric charge) is fixed. The other is the canonical ensemble where the physical electric charge is fixed. In these two ensembles, the associated free energies are the Gibbs free energy and Helmholtz free energy, respectively. When the Gibbs free energy or Helmholtz free energy is negative, the black hole phase is dominant over the thermal AdS background phase. This case implies that the black hole is globally stable, or say, globally preferred. When the Gibbs

\footnotetext{
* e-mail address: cairg@itp.ac.cn; Rong-Gen_Cai@baylor.edu

$\dagger$ e-mail address: Anzhong_Wang@baylor.edu
} 
(Helmholtz) free energy changes its sign, the Hawking-Page phase transition appears between the AdS black hole and thermal AdS background [9, 11]. Here the thermal AdS background corresponds to the case with vanishing mass parameter and gauge charge, and the AdS background as the vacuum background is assumed when we calculate the free energies associated with AdS black hole solutions. Another important property of black hole thermodynamics is the local stability, which in fact indicates whether a black hole can be in thermal equilibrium with the thermal bath around the black hole. For example, it is well-known that a Schwarzschild black hole cannot be in thermal equilibrium within an infinite thermal bath. This is because the heat capacity of the Schwarzschild is negative. Namely, when the mass of the black hole increases, the temperature of the black hole decreases. We will also discuss the local stability of hyperbolic charged black holes, which is determined by heat capacities in different ensembles.

The organization of the paper is as follows. In the next section we start with the hyperbolic Reissner-Nordström (RN) black holes in arbitrary dimensions. There we stress how to rewrite the solution in the isotropic coordinates and how to appropriately choose parameters to parameterize the solution so that the parameters can cover the whole phase space, which acts as service to the consequent related discussions for the hyperbolic black holes in $\mathrm{D}=5$, 4 , and 7 dimensional gauged supergravities. For the hyperbolic RN black holes, we find that the Gibbs free energy is always negative, and the heat capacity with a given electric potential or a given electric charge is also always positive, but the Helmholtz free energy can change its sign. In Sec. III, IV and V, we discuss the hyperbolic black holes in $\mathrm{D}=5,4$ and 7 dimensional gauged supergravities, respectively. Due to the appearance of nontrivial scalar fields, the thermodynamic properties and stability of the black holes get changed from the case of hyperbolic RN black holes. We present our main conclusions and give some discussions in Sec. VI.

\section{HYPERBOLIC RN BLACK HOLES IN ARBITRARY DIMENSIONS}

Let us start from an $(n+2)$-dimensional AdS RN black hole

$$
d s^{2}=-f(r) d t^{2}+f(r)^{-1} d r^{2}+r^{2} d \Omega_{n}^{2}
$$

where $d \Omega_{n}^{2}$ denotes the line element of an unit $n$-dimensional sphere and the function $f$ is given by

$$
f(r)=1-\frac{m}{r^{n-1}}+\frac{\tilde{q}^{2}}{r^{2 n-2}}+\frac{r^{2}}{l^{2}},
$$

$m$ and $\tilde{q}$ are the mass parameter and electric charge of the black hole, respectively. $l^{2}$ is related to the $(n+2)$ dimensional cosmological constant via $\Lambda=-n(n+1) / 2 l^{2}$. For the solution (2.1), one has to have $m>0$, otherwise, the singularity at $r=0$ is naked. When $m=2|\tilde{q}|$, the solution turns out to be supersymmetric and the function $f$ can be written as

$$
f=\left(1-\frac{\tilde{q}}{r^{n-1}}\right)^{2}+\frac{r^{2}}{l^{2}} .
$$

Obviously the solution in this case does not describe an AdS black hole, but a naked singularity. Defining

$$
m=\mu+2 q, \quad \tilde{q}^{2}=q(\mu+q), \quad r^{n-1} \rightarrow r^{n-1}+q,
$$

the solution (2.1) can be rewritten in the isotropic coordinates as

$$
d s^{2}=-H^{-2} f(r) d t^{2}+H^{2 /(n-1)}\left(f(r)^{-1} d r^{2}+r^{2} d \Omega_{n}^{2}\right),
$$

where

$$
f(r)=1-\frac{\mu}{r^{n-1}}+\frac{r^{2}}{l^{2}} H^{2 n /(n-1)}, \quad H=1+\frac{q}{r^{n-1}} .
$$

In this form the supersymmetric limit is obtained by taking $\mu \rightarrow 0$ while keeping $q$ finite. Furthermore, if introducing the so-called boost parameter $\beta$

$$
q=\mu \sinh ^{2} \beta
$$

the harmonic function $H$ can be written as $H=1+\mu \sinh ^{2} \beta / r^{n-1}$, the physical electric charge of the solution $\tilde{q}=\mu \sinh \beta \cosh \beta$, and the supersymmetric limit corresponds to the case $\beta \rightarrow \infty$ and $\mu \rightarrow 0$ while keeping $q$ finite. The electric potential of the solution (2.5) is

$$
A_{t}=\frac{\tilde{q}}{r^{n-1}+q} d t=\frac{\mu \sinh \beta \cosh \beta}{r^{n-1}+\mu \sinh ^{2} \beta} d t .
$$


Note that in the Schwarzschild coordinates (2.1), two integration constants are $m$ and $\tilde{q}$, and they are independent of each other. In the isotropic coordinates (2.5), they are changed to $\mu$ and $q$, and they could be related to each other via the relation (2.7). In addition, in the solution (2.5), $\mu \geq 0$ ( and then $q \geq 0$ via (2.7)). Otherwise, the solution describes a naked singularity.

Now we turn to the hyperbolic charged AdS black hole, whose metric is [ $]$

$$
d s^{2}=-f(r) d t^{2}+f(r)^{-1} d r^{2}+r^{2} d \Sigma_{n}^{2}
$$

where

$$
f(r)=-1-\frac{m}{r^{n-1}}+\frac{\tilde{q}^{2}}{r^{2 n-2}}+\frac{r^{2}}{l^{2}},
$$

$m$ and $\tilde{q}$ are two integration constants, $d \Sigma_{n}^{2}$ stands for the line element for an $n$-dimensional hypersurface with negative constant curvature $-n(n-1)$. One can obtain a closed black hole horizon by acting on the hyperbolic space $\Sigma_{n}$ by discrete subgroup of the isometric group of the hyperbolic space, resulting in a higher genus closed hypersurface. In four dimensions, the authors of [7] have shown that a magnetic charged hyperbolic solution with $m=0$ and $\tilde{q}^{2}=l^{2} / 4$ is supersymmetric. In that case, the metric function can be written as

$$
f=\left(\frac{l}{2 r}-\frac{r}{l}\right)^{2} .
$$

Curiously this is an extremal black hole solution with vanishing Hawking temperature, although the mass parameter $m=0$ in this solution. In fact, it is now well-known that there are so-called "zero mass" and "negative mass" black holes when the horizon is a hyperbolic surface. For example, when $m=0$, the solution (2.9) still has two black hole horizons $r_{1,2}^{2}=l^{2}\left(1 \pm \sqrt{1-4 \tilde{q}^{2} / l^{2}}\right) / 2$ provided $4 \tilde{q}^{2} / l^{2}<1$ in the four dimensional case. In fact, for the solution (2.10) the black hole horizon is still present, even the mass parameter $m$ is negative down to

$$
m_{c}=-2 r_{c}^{n-1}\left(1-\frac{n r_{c}^{2}}{(n-1) l^{2}}\right)
$$

where

$$
\frac{r_{c}^{2}}{l^{2}}=\frac{n-1}{n+1}\left(1+\frac{\tilde{q}^{2}}{r_{c}^{2 n-2}}\right) .
$$

When $m=m_{c}$, the solution describes an extremal black hole with vanishing Hawking temperature. When $m<m_{c}$, the singularity at $r=0$ becomes naked. Therefore, for a given charge, $r_{c}$ given in (2.13) is the smallest black hole horizon. Despite the horizon structure, so far we have already seen the big difference between the AdS RN solution (2.1) and the hyperbolic charged AdS solution (2.9).

It is instructive to rewrite the hyperbolic black hole solution (2.9) in terms of isotropic coordinates like in (2.5). Defining

$$
m=\mu-2 q, \quad \tilde{q}^{2}=q(\mu-q), \quad r^{n-1} \rightarrow r^{n-1}+q,
$$

we then have

$$
d s^{2}=-H^{-2} f(r) d t^{2}+H^{2 /(n-1)}\left(f(r)^{-1} d r^{2}+r^{2} d \Sigma_{n}^{2}\right)
$$

where

$$
f(r)=-1-\frac{\mu}{r^{n-1}}+\frac{r^{2}}{l^{2}} H^{2 n /(n-1)}, \quad H=1+\frac{q}{r^{n-1}}
$$

In these coordinates, we can see that both signs (positive and negative) of $\mu$ are allowed to have black hole horizon. (1) when $\mu>0$, one has to have $0<q<\mu$ in order to keep the electric charge squared $\tilde{q}^{2}$ positive. (2) When $\mu<0$, instead one has $\mu<q<0$. Combining these two cases, we find that it is appropriate to introduce a parameter $\alpha$ to parameterize the charge $q$ as

$$
q=\mu \sin ^{2} \alpha
$$


One then has $H=1+\mu \sin ^{2} \alpha / r^{n-1}$, the physical electric charge of the solution $\tilde{q}=\mu \sin \alpha \cos \alpha$, and the corresponding electric potential

$$
A_{t}=\frac{\mu \sin \alpha \cos \alpha}{r^{n-1}+\mu \sin ^{2} \alpha} d t=\frac{\tilde{q}}{r^{n-1}+q} d t .
$$

Note that when $q<0$, the original singularity ar $r=0$ in the coordinates $(2.9)$ is moved to $r=|q|$ in the coordinates (2.15). Furthermore, the supersymmetric solution (2.11) is achieved by taking $\sin ^{2} \alpha=1 / 2$ in the coordinates (2.15).

Note that $m$ and $\tilde{q}$ in (2.10) are two integration constants which are independently variable in the range $(-\infty, \infty)$. In the isotropic coordinates (2.15), these two integration constants are mapped to $\mu$ and $q$. From (2.17), however, one can see $0 \leq|q| \leq|\mu|$. Namely, given a $\mu$, one cannot get an arbitrary large $q$. Therefore, $\mu$ and $q$ through (2.14) does not cover the whole phase space given by $m$ and $\tilde{q}$, although in that case the expressions in (2.16) have very similar forms as those (2.6) of the AdS RN black hole. To remedy this difficulty, we find that the parameters $m$ and $q$ are good to do that. In this case, one has

$$
\begin{aligned}
& \tilde{q}^{2}=q(m+q) ; \\
& f(r)=-1-\frac{m+2 q}{r^{n-1}}+\frac{r^{2}}{l^{2}} H^{2 n /(n-1)}, \quad H=1+\frac{q}{r^{n-1}} .
\end{aligned}
$$

Furthermore, instead of (2.17), we can introduce a parameter $\beta$ to parameterize the charge $q$ as $q=m \sinh ^{2} \beta$ with $0 \leq \beta<\infty$. The physical electric charge becomes $\tilde{q}^{2}=m^{2} \sinh ^{2} \beta \cosh ^{2} \beta$. Thus, we see that $m$ and $q$ indeed cover the whole phase space as $m$ and $\tilde{q}$ in (2.9).

However, it turns out that it is more convenient to use the Schwarzschild coordinates (2.9) to discuss the thermodynamic properties and stability of the hyperbolic black hole. In terms of horizon radius $r_{+}$, determined by $\left.f(r)\right|_{r=r_{+}}=0$, for the hyperbolic black hole (2.9), the mass $M$, Hawking temperature $T$, entropy $S$ and chemical potential $\phi$ which is conjugate to the electric charge $\tilde{q}$ are found to be

$$
\begin{aligned}
& M=\frac{n V_{n}}{16 \pi G} m=\frac{n V_{n} r_{+}^{n-1}}{16 \pi G}\left(-1+\frac{\tilde{q}^{2}}{r_{+}^{2 n-2}}+\frac{r_{+}^{2}}{l^{2}}\right), \\
& T=\frac{(n-1)}{4 \pi r_{+}}\left(-1-\frac{\tilde{q}^{2}}{r_{+}^{2 n-2}}+\frac{n+1}{n-1} \frac{r_{+}^{2}}{l^{2}}\right), \\
& S=\frac{V_{n}}{4 G} r_{+}^{n}, \\
& \phi=\frac{n V_{n}}{16 \pi G} \frac{2 \tilde{q}}{r_{+}^{n-1}},
\end{aligned}
$$

where $V_{n}$ is the volume of the unit hyperbolic surface $\Sigma_{n}$. It can be checked that these quantities obey the first law of black hole thermodynamics

$$
d M=T d S+\phi d \tilde{q} .
$$

According to the AdS/CFT correspondence, the dual CFT resides on the boundary of the AdS space, whose metric can be determined from the bulk metric up to a conformal factor,

$$
d s^{2}=-d t^{2}+l^{2} d \Sigma_{n}^{2}
$$

The thermodynamics of the hyperbolic black hole can be mapped to that of the dual CFT residing on (2.25). From these thermodynamic quantities (2.20)-(2.23), we find that they satisfy the Cardy-Verlinde-like formula

$$
S=\frac{2 \pi l}{n} \sqrt{\left|E_{c}\right|\left(2\left(M-E_{q}\right)-E_{c}\right)}
$$

where $E_{c}=-n V_{n} r_{+}^{n-1} / 8 \pi G$ is the non-extensive part of energy, namely the Casimir energy, and $E_{q}=\phi \tilde{q} / 2$ is the energy of electromagnetic field outside the black hole. Thus we generalize the discussion made for neutral hyperbolic black hole [8] to the R-charged case.

Next we discuss thermodynamic stability and phase structure of the solution. In the grand canonical ensemble, we will fix the chemical potential conjugate to the electric charge 17. The Euclidean action $\mathcal{I}$ of the black hole solution has a relation to the Gibbs free energy $\mathcal{G}$ through $\mathcal{I}=\mathcal{G} / T[\underline{3}, 17]$. Therefore to see the global stability of the 
hyperbolic black hole as a thermodynamic system, we can calculate the Gibbs free energy, which is $\mathcal{G}=M-T S-\phi \tilde{q}$ by definition,

$$
\mathcal{G}=r_{+}^{n-1}\left(-1-\left(\frac{8 \pi G}{n V_{n}} \phi\right)^{2}-\frac{r_{+}^{2}}{l^{2}}\right) \frac{V_{n}}{16 \pi G}
$$

This Gibbs free energy is always negative, the hyperbolic black hole phase is therefore globally preferred, and the Hawking-Page transition will not happen here. Here some remarks are in order. Although the relation $\mathcal{G}=T \mathcal{I}$ between the Gibbs free energy $\mathcal{G}$ and the (reduced) Euclidean action $\mathcal{I}$ of black holes always holds (see Refs. 3, 17] and references therein), some subtleties exist, which concern the understanding of the Euclidean action and free energy. When using the relation $\mathcal{G}=T \mathcal{I}$ and the definition of free energy $\mathcal{G}=M-T S-\phi \tilde{q}$ here, we have taken the vacuum with $m=0$ and $\tilde{q}=0$ as the reference background, as in [3, 17]. In contrast to the cases of $k=0$ and $k=-1$, there the vacuum backgrounds are pure AdS spaces, not a black hole, the vacuum background of $k=-1$ is a back hole with horizon $r_{+}=l$, whose free energy is $\mathcal{G}_{0}=-l^{n-1} V_{n} /(8 \pi G)$. Subtracting this contribution, the Euclidean action should be understood as $\mathcal{I}=\left(\mathcal{G}-\mathcal{G}_{0}\right) T$. The action is always negative provided $r_{+}>l$, and is zero for the vacuum background. On the other hand, if taking the extremal black hole (2.12) as the reference background, as in [4], the black hole mass $M$ should be replaced by $M-M_{c}$, where $M_{c}=n V_{n} m_{c} / 16 \pi G$ with $m_{c}$ given by (2.12). It is easy to show that the Gibbs free energy $\mathcal{G}=\left(M-M_{c}\right)-T S-\phi \tilde{q}$ is always negative and approaches to zero for the extremal black hole. This is a natural generalization of the neutral hyperbolic black hole case discussed in [4].

In the grand canonical ensemble, the local stability condition of a thermodynamic system can be determined by the negative definiteness of the Hessian matrix. The Hessian matrix is arranged as the second derivatives of entropy with some extensive quantities. In our case, the extensive quantities are the black hole mass and charge. This approach is equivalent to calculating the heat capacity with the fixed chemical potential. The heat capacity of the hyperbolic black hole with a fixed electric potential (chemical potential) $\phi$ is given by

$$
\begin{aligned}
C_{\phi} & \equiv T\left(\frac{\partial S}{\partial T}\right)_{\phi} \\
& =\frac{n \pi V_{n} T r_{+}^{n+1}}{(n-1) G}\left(1+\frac{n+1}{n-1} \frac{r_{+}^{2}}{l^{2}}+2\left(\frac{8 \pi G}{n V_{n}} \phi\right)^{2}\right)^{-1} .
\end{aligned}
$$

One immediately sees that the heat capacity is always positive, which indicates the stability of the black hole in all range of temperature.

In the canonical ensemble, we will fix the electric charge of the system [17]. In this case, the Euclidean action of the solution will have a relation to the Helmholtz free energy as $\mathcal{I}=\mathcal{F} / T[3$, 17], here $\mathcal{F}=M-T S$. Then for the present hyperbolic black holes, we have

$$
\mathcal{F}=r_{+}^{n-1}\left(-1-\frac{r_{+}^{2}}{l^{2}}+(2 n-1) \frac{\tilde{q}^{2}}{r_{+}^{2 n-2}}\right) \frac{V_{n}}{16 \pi G}
$$

This indicates that when $\tilde{q}^{2}>\tilde{q}_{c}^{2}$, where

$$
\tilde{q}_{c}^{2}=\frac{r_{+}^{2 n-2}}{2 n-1}\left(1+\frac{r_{+}^{2}}{l^{2}}\right)
$$

the free energy is positive; otherwise it is negative. This implies that in the canonical ensemble with a fixed charge, when the temperature $T<T_{c}$, where

$$
T_{c}=\frac{(n-1)}{4 \pi r_{+}}\left(-1-\frac{\tilde{q}_{c}^{2}}{r_{+}^{2 n-2}}+\frac{n+1}{n-1} \frac{r_{+}^{2}}{l^{2}}\right),
$$

the black hole phase is not globally preferred, instead the thermal vacuum background is preferred. When $T>T_{c}$, the hyperbolic black hole phase is globally preferred. Across the temperature $T_{c}$, a Hawking-Page phase transition happens. In Fig. 1, we plot the physical charge squared versus horizon radius. The curve cross the horizon axe at $l / \sqrt{2} \approx 0.71 l$ denotes the extremal black holes with vanishing temperature. The region on the right side of the curve is physically allowed one with nonvanishing temperature. The other curve denotes the black hole solution having zero Helmholtz free energy. On the left (right) side of the curve the free energy is positive (negative). From the figure 


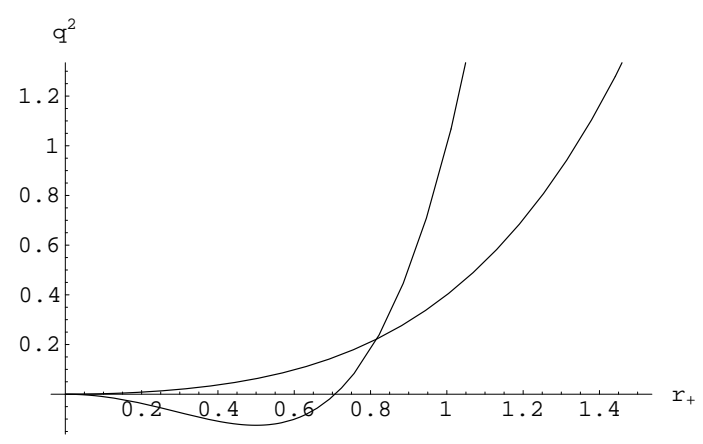

FIG. 1: The curves denote the charge squared $\tilde{q}^{2} / l^{4}$ versus the horizon radius $r_{+} / l$ for the case of $n=3$. In the region on the common right of the two curves the black hole phase is globally stable in the canonical ensemble. The curve across the horizon axe at $l / \sqrt{2} \approx 0.71 l$ denotes the extremal black holes with vanishing temperature.

one can see that the smallest horizon of black hole is $l / \sqrt{2}$. The region with negative $\tilde{q}^{2}$ should be excluded in the physical phase space.

As in the grand canonical ensemble, the Helmholtz free energy and then the Euclidean action are also dependent of the choice of the reference background. It should be emphasized here that as we calculate the Helmholtz free energy (2.29), the background is taken as the solution (2.9) with $m=\tilde{q}=0$, namely the so-called zero mass black hole. This might not be appropriate since the background has a vanishing electric charge, which does not satisfy the requirement of canonical ensemble. One of the choices as the background vacuums is to take the solution with $m=0$ and $\tilde{q}^{2}=\tilde{q}_{c}^{2}$. In this case, the background is also a black hole spacetime. The other is to take the solution (2.9) with mass $m=m_{c}$ in (2.12). In this case, the background is an extremal black hole. In either case, we have to recalculate the Euclidean action in order to see the globally stability. However, we expect that the main conclusions remain valid. We do not to stress here the difference arising from different vacuum backgrounds. For related discussion see [6].

The heat capacity of black hole with a fixed electric charge is found to be

$$
\begin{aligned}
C_{\tilde{q}} & \equiv T\left(\frac{\partial S}{\partial T}\right)_{\tilde{q}} \\
& =\frac{n \pi V_{n} T r_{+}^{n+1}}{(n-1) G}\left(1+\frac{n+1}{n-1} \frac{r_{+}^{2}}{l^{2}}+\frac{(2 n-1) \tilde{q}^{2}}{r_{+}^{2 n-2}}\right)^{-1} .
\end{aligned}
$$

This heat capacity, as the case in the grand canonical ensemble, is always positive. As a result, the black hole is always locally thermodynamically stable.

\section{HYPERBOLIC BLACK HOLES IN $D=5$ GAUGED SUPERGRAVITY}

The R-charged black holes in $\mathrm{D}=5, \mathrm{~N}=8$ gauged supergravity have been found in 18 as a special case (STU model) of the solutions of equations of motion of the $\mathrm{D}=5, \mathrm{~N}=2$ gauged supergravity (see also [19]). The black hole solution has the form

$$
\begin{aligned}
& d s^{2}=-\left(H_{1} H_{2} H_{3}\right)^{-2 / 3} f d t^{2}+\left(H_{1} H_{2} H_{3}\right)^{1 / 3}\left(f d r^{2}+r^{2} d \Omega_{3, k}^{2}\right), \\
& X_{i}=H_{i}^{-1}\left(H_{1} H_{2} H_{3}\right)^{1 / 3}, \quad A^{i}=\sqrt{k}\left(1-H_{i}^{-1}\right) \operatorname{coth} \beta_{i} d t
\end{aligned}
$$

where

$$
f=k-\frac{\mu}{r^{2}}+r^{2} l^{-2} H_{1} H_{2} H_{3}, \quad H_{i}=1+\frac{\mu \sinh ^{2} \beta_{i}}{k r^{2}}, \quad i=1,2,3
$$

where $k$ can be 1,0 or -1 , corresponding to the foliating surfaces of transverse space being $S^{3}, T^{3}$ or $H^{3}$ with unit metric $d \Omega_{3, k}^{2}, \mu$ is the mass parameter and $\beta_{i}$ are three boost parameters. When $k=1$ or $k=0$, the supersymmetric limit of the solution (3.1) is obtained via taking $\beta_{i} \rightarrow \infty$ and $\mu \rightarrow 0$, while keeping $\mu \sinh ^{2} \beta_{i}$ finite. Note that when $k=0$, one has to rescale $\sinh ^{2} \beta_{i} \rightarrow k \sinh ^{2} \beta_{i}$, and then send $k$ to zero, resulting in the gauge potential $A^{i}=\left(1-H_{i}^{-1}\right) d t / \sinh \beta_{i}$. Therefore, the supersymmetric solution has vanishing gauge potential in the case of $k=0$. 
The thermodynamics of cases $k=1$ and $k=0$ has been discussed in [14]. Here we focus on the case $k=-1$, namely the case where the horizon surface is a hyperbolic 3-space with constant negative curvature. In this case, the supersymmetric limit of the solution cannot be obtained as the cases of $k=1$ and $k=0$. So far it has not yet been clear whether the solution has the supersymmetric limit, or under what condition the solution is supersymmetric. In particular, let us note that in the solution (3.1), if one defines $q_{i}=\mu \sinh ^{2} \beta_{i} / k$, the physical electric charges are

$$
\tilde{q}_{i}=\sqrt{k} \mu \sinh \beta_{i} \cosh \beta_{i} .
$$

When $k=-1$, one can see from (3.2) that the electric potentials $A^{i}$ are pure imaginary if keeping $\beta_{i}$ real. This indicates that the parametrization used in (3.2) and (3.3) is not suitable for the case of $k=-1$. Note that the solution is written down in the isotropic coordinates and it will reduce to the $\mathrm{D}=5$ hyperbolic AdS RN black hole in (2.9) as all three charges are equal in (3.1). According to the experience in the previous section, we find that the suitable parametrization can be obtained by the replacement

$$
\beta_{i} \rightarrow 1 \alpha_{i}
$$

when $k=-1$. Here 1 denotes the unit pure imaginary number. Upon this replacement, we have

$$
H_{i}=1+\frac{q_{i}}{r^{2}}, \quad A^{i}=\frac{\tilde{q}_{i}}{r^{2}+q_{i}} d t
$$

where

$$
q_{i}=\mu \sin ^{2} \alpha_{i}, \quad \tilde{q}_{i}^{2}=q_{i}\left(\mu-q_{i}\right) .
$$

As stressed in the previous section, in fact, this parametrization is also unsuitable due to the restriction $0 \leq|q| \leq|\mu|$. We find, however, that when the solution has only an electric charge parameter, which includes three cases: (i) three charges are equal, $q_{1}=q_{2}=q_{3}=q$; (ii) two charges are equal, the third vanishes, say, $q_{1}=q_{2}=q$ and $q_{3}=0$; (iii) one charge does not vanish, say $q_{1}=q$, and the other two vanish, one can remedy the difficulty $\left|q_{i}\right| \leq \mid \mu$ in (3.7). Introducing

$$
\mu=m+2 q, \quad \tilde{q}^{2}=q(m+q),
$$

we then have

$$
f=-1-\frac{m+2 q}{r^{2}}+r^{2} l^{-2} H_{1} H_{2} H_{3}, \quad H_{i}=1+\frac{q}{r^{2}},
$$

where $H_{1} H_{2} H_{3}$ depends on the number of nonvanishing charges. In that case, $m$ and $q$ become two parameters varying independently in the range $(-\infty, \infty)$, and $q$ can be parameterized via $q=m \sinh ^{2} \beta$. Note that in this parametrization, one has $q>0(<0)$ if $m>0(<0)$.

Using the background subtraction approach [20], the mass of the hyperbolic black hole can be calculated following the case of $k=1$ [18]. Rewriting the solution in terms of Schwarzschild coordinates

$$
d s^{2}=-e^{-2 V} d t^{2}+e^{2 W} d R^{2}+R^{2} d \Omega_{3,-1}^{2},
$$

where $R^{2}=r^{2}\left(H_{1} H_{2} H_{3}\right)^{1 / 3}$ and

$$
e^{-2 V}=f\left(H_{1} H_{2} H_{3}\right)^{-2 / 3}, \quad e^{2 W}=f^{-1}\left(H_{1} H_{2} H_{3}\right)^{1 / 3}\left(\frac{\partial r}{\partial R}\right)^{2},
$$

one can find the mass of the solution using the formula

$$
M=-\frac{1}{8 \pi G} \int_{\partial \mathcal{M}} N\left(K-K_{0}\right),
$$

where $N=e^{-V}$ is the norm of the time-like Killing vector and $K$ is the extrinsic curvature of induced boundary. $K_{0}$ is the same as $K$, but for the vacuum background, namely the solution with $\mu=0$ and $q_{i}=0$. After calculation, it turns out that the mass of the hyperbolic black hole is

$$
M=\frac{V_{3}}{8 \pi G}\left(\frac{3}{2} \mu-\sum_{i} q_{i}\right) .
$$


It is interesting to compare with the cases of $k=1$ and $k=0$, where the mass is $M=V_{3}\left(3 \mu / 2+\sum q_{i}\right) / 8 \pi G$ for $k=1$, while $M=3 V_{3} \mu / 16 \pi G$ for $k=0$. Here $V_{3}$ is the volume of $\Omega_{3, k}$.

The event horizon of the hyperbolic black hole is determined by $\left.f(r)\right|_{r=r_{+}}=0$. In terms of the horizon radius, the mass parameter $\mu$ can be expressed as

$$
\mu=r_{+}^{2}\left(-1+\Pi_{i} \rho_{i}^{2} / r_{+}^{4} l^{2}\right), \quad \rho_{i}^{2}=r_{+}^{2}+q_{i}
$$

The Hawking temperature $T$, entropy $S$ and chemical potential $\phi_{i}$ conjugate to the electric charge $\tilde{q}_{i}$ are

$$
\begin{aligned}
T & =\frac{r_{+}^{2}}{2 \pi \Pi_{i} \rho_{i}}\left(-1-\frac{\Pi_{i} \rho_{i}^{2}}{l^{2} r_{+}^{4}}+\frac{\Pi_{i} \rho_{i}^{2}}{l^{2} r_{+}^{2}} \sum_{i} \frac{1}{\rho_{i}^{2}}\right), \\
S & =\frac{V_{3}}{4 G} \Pi_{i} \rho_{i}, \\
\phi_{i} & =\frac{V_{3}}{8 \pi G} \frac{\tilde{q}_{i}}{\rho_{i}^{2}},
\end{aligned}
$$

respectively. In terms of the horizon radius $r_{+}$, the expression of the mass of black hole is

$$
M=\frac{3 V_{3}}{16 \pi G}\left(r_{+}^{2}+\frac{\Pi_{i} \rho_{i}^{2}}{l^{2} r_{+}^{2}}-\frac{2}{3} \sum_{i} \rho_{i}^{2}\right) .
$$

It is easy to show that the first law of thermodynamics holds here, $d M=T d S+\sum_{i} \phi_{i} d \tilde{q}_{i}$. For this black hole, the Gibbs free energy, defined as $\mathcal{G}=M-T S-\sum_{i} \phi_{i} \tilde{q}$, is found to be

$$
\mathcal{G}=\frac{V_{3}}{16 \pi G}\left(-r_{+}^{2}-\frac{\Pi_{i} \rho_{i}^{2}}{l^{2} r_{+}^{2}}\right)
$$

which is always negative. The negative definiteness indicates that the Hawking-Page phase transition will not occur. The black hole phase always dominates in the whole range of temperature and chemical potential. Here it also should be stressed that the same discussions made in the previous section for the case of hyperbolic RN black holes are applicable. The reference background is a five dimensional "zero mass" black hole with $\mu=q_{i}=0$, whose free energy is $\mathcal{G}_{0}=-l^{2} V_{3} / 8 \pi G$. The reduced action should be understood as $\mathcal{I}=\left(\mathcal{G}-\mathcal{G}_{0}\right) / T$, which is negative provided $r_{+}>l$. If taking the extremal black hole with vanishing Hawking temperature as the vacuum background, the black hole mass $M$ should be replaced by $M-M_{c}$, once again. In this case, the resulting Euclidean action is always negative.

The Helmholtz free energy, defined as $\mathcal{F}=M-T S$, is

$$
\mathcal{F}=\frac{V_{3}}{16 \pi G}\left(5 r_{+}^{2}+5 \frac{\Pi_{i} \rho_{i}^{2}}{l^{2} r_{+}^{2}}-2 \sum_{i} \rho_{i}^{2}-2 \frac{\Pi_{i} \rho_{i}^{2}}{l^{2}} \sum_{i} \frac{1}{\rho_{i}^{2}}\right) .
$$

This free energy can be negative or positive. To see clearly this, let us consider a single charge case. Namely, one of three charges of the solution, say, $q_{1}=q \neq 0$, and other two $q_{2}=q_{3}=0$. In this case, the equation $f(r)=0$ has the following roots

$$
r_{1,2}^{2}=\frac{l^{2}}{2}\left(\left(1-\frac{q}{l^{2}}\right) \pm \sqrt{\left(1-\frac{q}{l^{2}}\right)^{2}+4 \frac{\mu}{l^{2}}}\right)
$$

From the above, we see some interesting features of the black hole. (1) When $q<l^{2}$, the hyperbolic black hole has two horizons if $-l^{2}\left(1-q / l^{2}\right)^{2} / 4<\mu<0$, while it has only one horizon if $\mu>0$. When $\mu=-l^{2}\left(1-q / l^{2}\right)^{2} / 4$, these two horizons coincides with each other. This case corresponds to the extremal black holes with vanishing Hawking temperature. (2) When $q>l^{2}$, the solution has one black hole horizon only if $\mu>0$. (3) When $q=l^{2}$, the black hole has a horizon with $r_{+}^{2}=l \sqrt{\mu}$. (4) When $q=0$, the solution goes back to the case of neutral hyperbolic black hole.

(5) When $\mu=0$, the black hole has also two horizons: one of them $r_{+}=0$, the other is $r_{+}^{2}=l^{2}-q$ if $q<l^{2}$.

In the single charge case, the Hawking temperature is

$$
T=\frac{1}{2 \pi \rho}\left(-1+\frac{\rho^{2}}{l^{2}}+\frac{r_{+}^{2}}{l^{2}}\right), \quad \rho^{2}=r_{+}^{2}+q,
$$


and the Helmholtz free energy reduces to

$$
\mathcal{F}=\frac{V_{3}}{16 \pi G}\left(-r_{+}^{2}-2 q-\frac{r_{+}^{4}}{l^{2}}+\frac{r_{+}^{2}}{l^{2}} q\right)
$$

Therefore, when $r_{+}^{2} / l^{2} \leq 2$, the free energy is always negative, while $q<r^{2}\left(1+r^{2} / l^{2}\right) /\left(r^{2} / l^{2}-2\right)$ as $r_{+}^{2} / l^{2}>2$. In Fig. 2 the region with negative free energy is plotted between the two curves. The curve starting at $q=1$ and $r_{+}=0$ and ending at $q=0$ and $r_{+} / l=1 / \sqrt{2} \approx 0.71$ denotes extremal black holes. So the region below this curve should be excluded in the physical phase space. The above curve in Fig. 2 denotes the black holes with zero free energy. Therefore, a Hawking-Page phase transition happens across that curve. Note that the free energy depends on the choice of vacuum background. Here the vacuum background is taken as the "zero mass" black hole with $\mu=q=0$. Other choices are of course interest to further investigate, as the case of hyperbolic RN black holes.

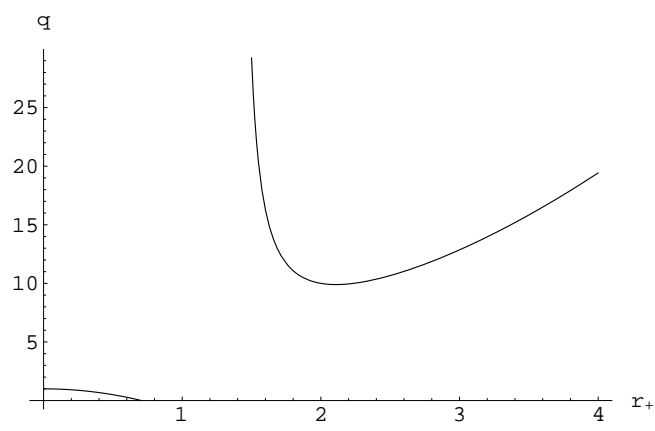

FIG. 2: The curves denote the charge $q / l^{2}$ versus the horizon radius $r_{+} / l$. The curve starting from $q=1$ and $r_{+}=0$ and ending at $q=0$ and $r_{+} / l=1 / \sqrt{2}$ denotes extremal black holes with vanishing temperature. The above curve stands for black holes with zero free energy.

The local stability of black hole thermodynamics is determined by heat capacity. The heat capacity with a fixed charge is

$$
C_{\tilde{q}}=\frac{\pi V_{3} T}{2 G} \frac{l^{2}\left(r_{+}^{2}+q\right)\left(3 r_{+}^{2}\left(r_{+}^{2}+q\right)-l^{2}\left(3 r_{+}^{2}+4 q\right)\right)}{2 r_{+}^{4}+5 q r_{+}^{2}-l^{2}\left(r_{+}^{2}+6 q\right)-q^{2}-l^{4}} .
$$

The heat capacity with a fixed potential turns out to be

$$
C_{\phi}=\frac{\pi V_{3} T}{2 G} \frac{l^{2} r_{+}^{2}\left(r_{+}^{2}+q\right)\left(3 r_{+}^{2}-3 l^{2}-q\right)}{2 r_{+}^{4}+q r_{+}^{2}-l^{2}\left(2 q+r_{+}^{2}\right)-q^{2}-l^{4}}
$$

In Fig. 3 and 4 we plot the regions where the heat capacities $C_{\tilde{q}}$ and $C_{\phi}$ are negative, respectively.

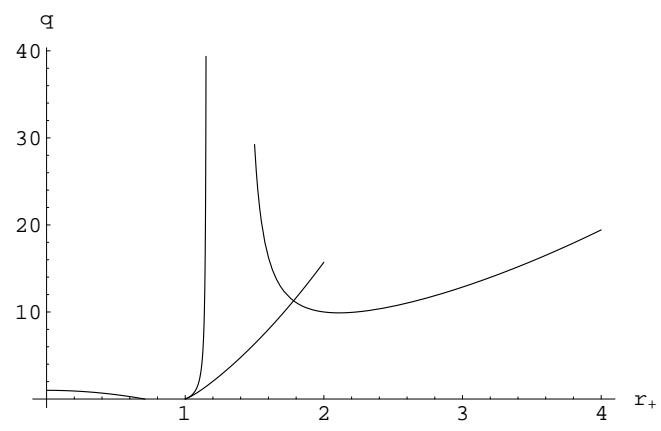

FIG. 3: The curves denote the charge $q / l^{2}$ versus the horizon radius $r_{+} / l$. The two curves starting at $q=0$ and $r_{+} / l=1$ enclose a region where the heat capacity with a fixed charge $C_{\tilde{q}}$ is negative. In other physical regions $C_{\tilde{q}}$ is positive. 


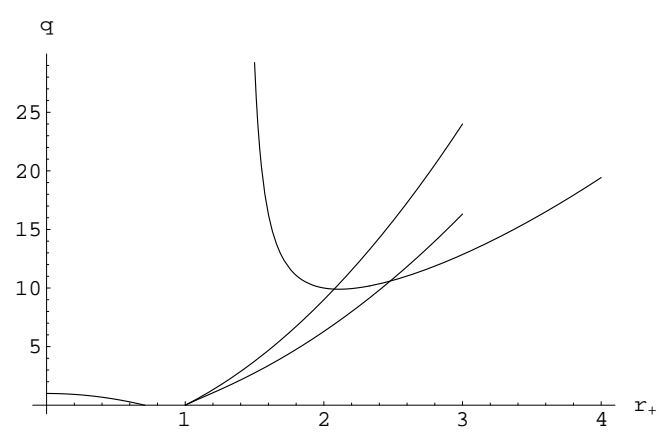

FIG. 4: The curves denote the charge $q / l^{2}$ versus the horizon radius $r_{+} / l$. The two curves starting at $q=0$ and $r_{+} / l=1$ enclose a region where the heat capacity with a fixed potential $C_{\phi}$ is negative. In other physical regions $C_{\phi}$ is positive.

\section{HYPERBOLIC BLACK HOLES IN $D=4$ GAUGED SUPERGRAVITY}

The black holes in $\mathrm{D}=4, \mathrm{~N}=8$ gauged supergravity has been found in [21, 22] (see also [19]),

$$
\begin{aligned}
& d s^{2}=-\left(H_{1} H_{2} H_{3} H_{4}\right)^{-1 / 2} f d t^{2}+\left(H_{1} H_{2} H_{3} H_{4}\right)^{1 / 2}\left(f d r^{2}+r^{2} d \Omega_{2, k}^{2}\right), \\
& X_{i}=H_{i}^{-1}\left(H_{1} H_{2} H_{3} H_{4}\right)^{1 / 4}, \quad A^{i}=\sqrt{k}\left(1-H_{i}^{-1}\right) \operatorname{coth} \beta_{i} d t
\end{aligned}
$$

where

$$
f=k-\frac{\mu}{r}+\frac{r^{2}}{l^{2}} H_{1} H_{2} H_{3} H_{4}, \quad H_{i}=1+\frac{\mu \sinh ^{2} \beta_{i}}{k r}, \quad i=1,2,3,4 .
$$

As the case of $\mathrm{D}=5$, the supersymmetric limit of the solution is obtained by taking $\mu \rightarrow 0$ and $\beta_{i} \rightarrow \infty$ while keeping $\mu \sinh ^{2} \beta_{i}$ finite. But the supersymmetric limit can be obtained only for the cases $k=1$ and $k=0$ (In the case of $k=0$, one has to first take $\sinh ^{2} \beta_{i} \rightarrow k \sinh ^{2} \beta_{i}$ as the case of $D=5$ ). When $k=-1$, we have to make the replacement $\beta_{i} \rightarrow 1 \alpha_{i}$, and then obtain a real gauge potential. In this case, one has $q_{i}=\mu \sin ^{2} \alpha_{i}$ and the physical charge $\tilde{q}_{i}=\mu \sin \alpha_{i} \cos \alpha_{i}$. Once again, $\mu$ and $\alpha_{i}$ are not good parameters because of $0 \leq\left|q_{i}\right| \leq|\mu|$. As the case of $D=5$, when the solution has only a charge parameter (which includes four cases: all four charges are equal; three charges are equal and one vanishes; two charges are equal and the other two vanish; and one of charges does not vanishes and the other three charges are zero), we can take $m$ and $\beta$ as two good parameters: $q=m \sinh ^{2} \beta$ and $\mu=m+2 q$. And in this case, one has the physical charge $\tilde{q}^{2}=q(m+q)=m^{2} \sinh ^{2} \beta \cosh ^{2} \beta$. In this way, $m$ and $q$ can vary independently in the range $(-\infty, \infty)$.

The horizon radius of the hyperbolic black hole is determined by the equation

$$
\mu=r_{+}\left(-1+\frac{1}{r_{+}^{2} l^{2}} \Pi_{i} \rho_{i}\right), \quad \rho_{i}=r_{+}+q_{i} .
$$

For the black hole, the associated mass $M$, Hawking temperature $T$, entropy $S$, and the chemical potential $\phi_{i}$ conjugate to the physical electric charge $\tilde{q}_{i}$ are

$$
\begin{aligned}
M & =\frac{V_{2}}{16 \pi G}\left(2 \mu-\sum_{i} q_{i}\right), \\
T & =\frac{r_{+}}{4 \pi \sqrt{\Pi_{i} \rho_{i}}}\left(-1-\frac{\Pi_{i} \rho_{i}}{l^{2} r_{+}^{2}}+\frac{\Pi_{i} \rho_{i}}{l^{2} r_{+}} \sum_{i} \frac{1}{\rho_{i}}\right), \\
S & =\frac{V_{2}}{4 G} \sqrt{\Pi_{i} \rho_{i}}, \\
\phi_{i} & =\frac{V_{2}}{16 \pi G} \frac{\tilde{q}_{i}}{\rho_{i}}
\end{aligned}
$$

respectively. They obey $d M=T d S+\sum_{i} \phi_{i} d \tilde{q}_{i}$. The Gibbs free energy reads

$$
\mathcal{G}=\frac{V_{2}}{16 \pi G}\left(-r_{+}-\frac{\Pi_{i} \rho_{i}}{l^{2} r_{+}}\right)
$$


which is always negative, while the Helmholtz free energy of the hyperbolic black hole is

$$
\mathcal{F}=\frac{V_{2}}{16 \pi G}\left(-r_{+}-\frac{\Pi_{i} \rho_{i}}{l^{2} r_{+}}-\sum_{i} q_{i}+\frac{\Pi_{i} \rho_{i}}{l^{2} r_{+}} \sum_{i} \frac{q_{i}}{\rho_{i}}\right) .
$$

As the case of $D=5$, the reference background is chosen as the "zero mass" black hole with $\mu=q_{i}=0$. The discussions of the reference background dependence of the free energies for the hyperbolic RN black holes are of course still valid for the $\mathrm{D}=4$ hyperbolic black holes. We do not repeat here.

For a single charge case, the free energy reduces to

$$
\mathcal{F}=\frac{V_{2}}{16 \pi G}\left(-r_{+}-\frac{r_{+}^{3}}{l^{2}}-q\right) .
$$

The heat capacities are

$$
C_{\tilde{q}}=\frac{\pi V_{2} T}{G} \frac{l^{2} r_{+}^{2}\left(r_{+}+q\right)\left(2 r_{+}^{2}\left(r_{+}+q\right)-l^{2}\left(2 r_{+}+3 q\right)\right)}{3 r_{+}^{4}\left(r_{+}+2 q\right)-r_{+} l^{2}\left(2 q^{2}+7 q r_{+}+2 r_{+}^{2}\right)-l^{4}\left(r_{+}+q\right)},
$$

for a given electric charge, and

$$
C_{\phi}=\frac{\pi V_{2} T}{G} \frac{\left.l^{2} r_{+}^{2}\left(r_{+}+q\right)\left(\left(2 r_{+}-q\right) r_{+}-2 l^{2}\right)\right)}{r_{+}^{2}\left(3 r_{+}^{2}+q r_{+}-2 q^{2}\right)-l^{2} r_{+}\left(3 q+2 r_{+}\right)-l^{4}},
$$

for a given gauge potential, where the Hawking temperature is

$$
T=\frac{1}{4 \pi \sqrt{r_{+} \rho}}\left(-1+\frac{2 r_{+} \rho}{l^{2}}+\frac{r_{+}^{2}}{l^{2}}\right), \quad \rho=r_{+}+q .
$$

We see that for a single charge case (say, $q_{1}=q$ and $q_{2}=q_{3}=q_{4}=0$ ), the Helmholtz free energy is also always negative. But to keep the positive definiteness of the Hawking temperature, one has $q>l^{2}\left(1-3 r_{+}^{2} / l^{2}\right) / 2 r_{+}^{2}$ when $0<r_{+}^{2} / l^{2}<1 / 3$ (while the temperature is always of positive definiteness when $r_{+}^{2} / l^{2}>1 / 3$ ). Therefore the HawkingPage phase transition will not occur in this case. The black hole phase is always globally dominant in both the grand canonical ensemble and the canonical ensemble. However, the hyperbolic black holes are not always locally thermodynamically stable. In Fig. 5 and 6 the thermodynamically unstable regions are plotted in the canonical ensemble and grand canonical ensemble, respectively. In these two figures, the curve starting from $q \rightarrow \infty$ and $r_{+}=0$ and ending at $q=0$ and $r_{+} / l=1 / \sqrt{3} \approx 0.58$ denotes extremal black holes, the region below that curve should be excluded in the physical phase space.

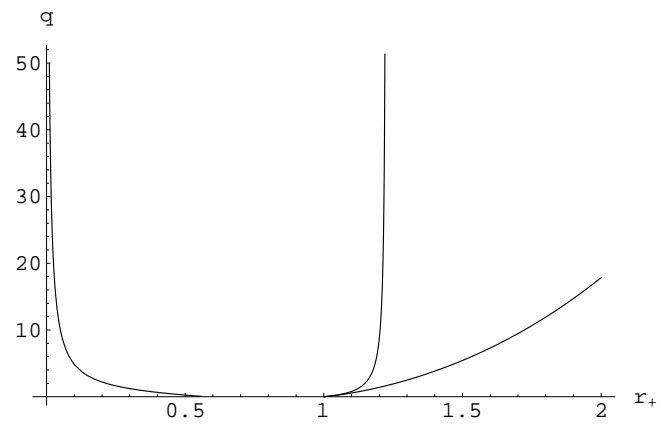

FIG. 5: The curves denote the charge $q / l$ versus the horizon radius $r_{+} / l$. The first curve from left to right denotes extremal black holes with vanishing temperature. The two curves starting at $q=0$ and $r_{+} / l=1$ enclose a region where $C_{\tilde{q}}<0$. In other physical regions $C_{\tilde{q}}>0$.

\section{HYPERBOLIC BLACK HOLES IN $D=7$ GAUGED SUPERGRAVITY}

The black hole solution in $\mathrm{D}=7, \mathrm{~N}=4$ gauged supergravity is [14] (see also [19])

$$
\begin{aligned}
& d s^{2}=-\left(H_{1} H_{2}\right)^{-4 / 5} f d t^{2}+\left(H_{1} H_{2}\right)^{1 / 5}\left(f d r^{2}+r^{2} d \Omega_{5, k}^{2}\right), \\
& X_{i}=H_{i}^{-1}\left(H_{1} H_{2}\right)^{2 / 5}, \quad A^{i}=\sqrt{k}\left(1-H_{i}^{-1}\right) d t,
\end{aligned}
$$




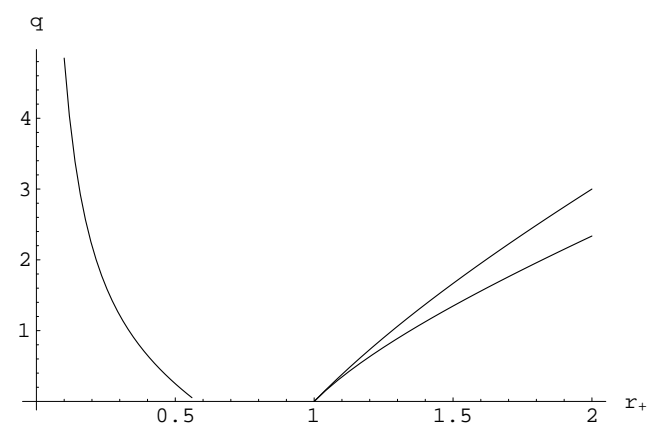

FIG. 6: The curves denote the charge $q / l$ versus the horizon radius $r_{+} / l$. The first curve from left to right denotes extremal black holes with vanishing temperature. The region enclosed by the two curves starting at $q=0$ and $r_{+} / l=1$ denotes black holes having negative heat capacity with a fixed potential. In other physical regions $C_{\phi}>0$.

where

$$
f=k-\frac{\mu}{r^{4}}+\frac{r^{2}}{l^{2}} H_{1} H_{2}, \quad H_{i}=1+\frac{\mu \sinh ^{2} \beta_{i}}{k r^{4}}, \quad i=1,2 .
$$

As the cases of $D=5$ and $D=4$, when $k=-1$, in order to get a real gauge potential, one can take $\beta_{i} \rightarrow 1 \alpha_{i}$. In this case, $q_{i}=\mu \sin ^{2} \alpha_{i}$ and $\tilde{q}_{i}^{2}=\mu^{2} \sin ^{2} \alpha_{i} \cos ^{2} \alpha_{i}$. Therefore $\mu$ and $\alpha_{i}$ are not good parameters as the cases of $D=5$ and $D=4$. When the solution has only one charge parameter, once again, we can remedy this difficulty by taking $m$ and $\beta$ as two parameters describing the solution, $q=m \sinh ^{2} \beta$ and $\mu=m+2 q$, and then the physical charge $\tilde{q}^{2}=m^{2} \sinh ^{2} \beta \cosh ^{2} \beta$. The single charge parameter case includes two cases: (1) the two charges are equal, $q_{i}=q_{2}=q ;(2)$ one of them vanishes, say $q_{1}=q$ and $q_{2}=0$.

For this hyperbolic black hole, we obtain the associated mass, Hawking temperature, entropy and chemical potential

$$
\begin{aligned}
M & =\frac{V_{5}}{4 \pi G}\left(\frac{5}{4} \mu-\sum_{i} q_{i}\right), \\
T & =\frac{r_{+}^{3}}{\pi \Pi_{i} \rho_{i}^{2}}\left(-1-\frac{\Pi_{i} \rho_{i}^{4}}{2 l^{2} r_{+}^{6}}+\frac{\Pi_{i} \rho_{i}^{4}}{l^{2} r_{+}^{2}} \sum_{i} \frac{1}{\rho_{i}^{4}}\right), \\
S & =\frac{V_{5}}{4 G} r_{+} \Pi_{i} \rho_{i}^{2}, \\
\phi_{i} & =\frac{V_{5}}{4 \pi G} \frac{\tilde{q}_{i}}{\rho_{i}^{4}},
\end{aligned}
$$

where the horizon is determined by the equation

$$
\mu=r_{+}^{4}\left(-1+\frac{1}{l^{2} r_{+}^{6}} \Pi_{i} \rho_{i}^{4}\right), \quad \rho_{i}^{4}=r_{+}^{4}+q_{i} .
$$

It is easy to show $d M=T d S+\sum_{i} \phi_{i} \tilde{q}_{i}$. The Gibbs free energy is found to be

$$
\mathcal{G}=\frac{V_{5}}{16 \pi G}\left(-r_{+}^{4}-\frac{\Pi_{i} \rho_{i}^{4}}{l^{2} r_{+}^{2}}\right),
$$

and the Helmholtz free energy

$$
\mathcal{F}=\frac{V_{5}}{16 \pi G}\left(-r_{+}^{4}-4 \sum_{i} q_{i}-\frac{4 r_{+}^{2}}{l^{2}} \Pi_{i} \rho_{i}^{4} \sum_{i} \frac{1}{\rho_{i}^{4}}+7 \frac{\Pi_{i} \rho_{i}^{4}}{l^{2} r_{+} 62}\right) .
$$

Once again, we see that the Gibbs free energy is always negative. As a result the Hawking-Page phase transition will not happen in the grand canonical ensemble, while in the canonical ensemble it is possible. Of course, the free energies and the Euclidean actions depend on the choice of reference background. Here as the case of hyperbolic RN black hole, a seven dimensional "zero mass" black hole has been taken. Similar discussions made for the hyperbolic $\mathrm{RN}$ black holes can be repeated. But we do not present them them here. 
For the single charge case, where one of charges vanishes, we have

$$
T=\frac{r_{+}}{\pi \rho^{2}}\left(-1+\frac{\rho^{4}}{2 l^{2} r_{+}^{2}}+\frac{r_{+}^{2}}{l^{2}}\right), \quad \rho^{4}=r_{+}^{4}+q,
$$

and the free energy reduces to

$$
\mathcal{F}=\frac{V_{5}}{16 \pi G}\left(-r_{+}^{4}-4 q-\frac{r_{+}^{6}}{l^{2}}+3 \frac{r_{+}^{2}}{l^{2}} q\right)
$$

In order to have $\mathcal{F} \leq 0$, one has to have $q<r_{+}^{4}\left(1+r_{+}^{2} / l^{2}\right) /\left(3 r_{+}^{2} / l^{2}-4\right)$ for $r_{+}^{2} / l^{2}>4 / 3$. When $r_{+}^{2} / l^{2}<4 / 3$, the free energy is always negative. On the other hand, the positive definiteness of the temperature has to be guaranteed. Therefore $q>r_{+}^{2} l^{2}\left(2-3 r_{+}^{2} / l^{2}\right)$ when $0 \leq r_{+} / l<\sqrt{6} / 3 \approx 0.82$. In Fig. 7 the curve standing for the extremal black holes and the curve having zero free energy are plotted. The region below the extremal black hole curve starting from $q=0$ and $r_{+}=0$ and ending at $q=0$ and $r_{+} / l=0.82$ should be excluded in the physical phase space. Note that for the sake of demonstration, the charge $q$ for extremal black holes is amplified by ten times in Fig. 7 and 8 and 9 .

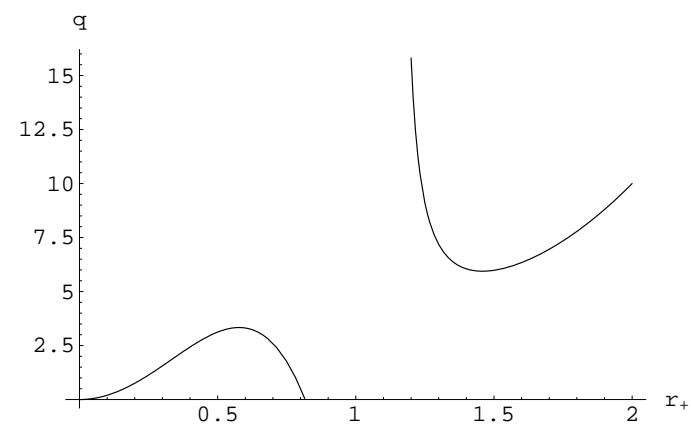

FIG. 7: The curves denote the charge $q / l^{4}$ versus the horizon radius $r_{+} / l$. The curves starting at $q=0$ and $r_{+}=0$ and ending at $q=0$ and $r_{+} / l=0.82$ denotes extremal black holes with vanishing Hawking temperature. The another curve stands for the black holes having zero Helmholtz free energy, in the region below this curve black holes have negative free energy. Note that for the sake of demonstration, the charge $q$ for extremal black holes is amplified by ten times here.

The heat capacities are

$$
C_{\tilde{q}}=\frac{\pi V_{5} T}{4 G} \frac{2 l^{2} r_{+}^{4}\left(r_{+}^{4}+q\right)\left(5 r_{+}^{2}\left(r_{+}^{4}+q\right)-l^{2}\left(5 r_{+}^{4}+6 q\right)\right)}{3 r_{+}^{2}\left(r_{+}^{8}+4 q r_{+}^{4}-q^{2}\right)-l^{2}\left(-2 q^{2}+17 q r_{+}^{4}+r_{+}^{8}\right)-l^{4}\left(2 r_{+}^{6}-4 q r_{+}^{2}\right)}
$$

for a fixed charge, and

$$
C_{\phi}=\frac{\pi V_{5} T}{4 G} \frac{2 l^{2} r_{+}^{4}\left(5 r_{+}^{8}-5 l^{2} r_{+}^{6}+4 q r_{+}^{4}-5 l^{2} q r_{+}^{2}-q^{2}\right)}{3 r_{+}^{8}-l^{2} r_{+}^{6}+2 q r_{+}^{4}-2 l^{4} r_{+}^{4}-3 l^{2} q r_{+}^{2}-q^{2}}
$$

for a fixed gauge potential, respectively. In Fig. 8, the region for the locally thermodynamically unstable black holes in the grand canonical ensemble is plotted. The narrow region between the two curves both starting at $q=0$ and $r_{+} / l=1$ has $C_{\phi}<0$.

On the other hand, in the canonical ensemble the hyperbolic black hole is locally thermodynamical stable in the regions as follows: (1) When $0<r_{+} / l<\sqrt{2 / 3} \approx 0.82$,

$$
\left(2-3 \frac{r_{+}^{2}}{l^{2}}\right) \frac{r_{+}^{2}}{l^{2}}<\frac{q}{l^{4}}<\frac{q_{c 1}}{l^{4}}
$$

where

$$
\frac{q_{c 1}}{l^{4}}=\frac{17 r_{+}^{4} / l^{4}-12 r_{+}^{6} / l^{6}-4 r_{+}^{2} / l^{2}+\sqrt{\left(12 r_{+}^{6} / l^{6}-17 r_{+}^{4} / l^{4}-4 r_{+}^{2} / l^{2}\right)^{2}-4\left(2-3 r_{+}^{2} / l^{2}\right)\left(3 r_{+}^{10} / l^{10}-r_{+}^{8} / l^{8}-2 r_{+}^{6} / l^{6}\right)}}{2\left(2-3 r_{+}^{2} / l^{2}\right)} .
$$




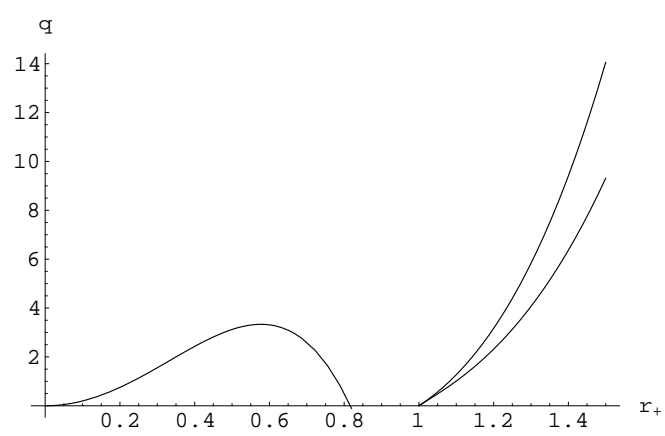

FIG. 8: The curves denote the charge $q / l^{4}$ versus the horizon radius $r_{+} / l$. In the region between the two curves starting at $q=0$ and $r_{+} / l=1$ one has $C_{\phi}<0$. In the other physical region, $C_{\phi}>0$.

(2) When $\sqrt{2 / 3}<r_{+} / l<1$, the black hole is always thermodynamical stable for any $q$. (3) When $1<r_{+} / l<$ $\sqrt{6 / 5} \approx 1.1$, the black hole is locally thermodynamically stable for $q>q_{2 c}$ or $q<q_{3 c}$. (4) When $r_{+} / l>\sqrt{6 / 5}$, one has to have $q<q_{3 c}$, where

$$
\frac{q_{2 c}}{l^{4}}=\frac{5 r_{+}^{4}\left(r_{+}^{2} / l^{2}\right)}{l^{4}\left(6-5 r_{+}^{2} / l^{2}\right)}
$$

and

$$
\frac{q_{c 3}}{l^{4}}=\frac{17 r_{+}^{4} / l^{4}-12 r_{+}^{6} / l^{6}-4 r_{+}^{2} / l^{2}-\sqrt{\left(12 r_{+}^{6} / l^{6}-17 r_{+}^{4} / l^{4}-4 r_{+}^{2} / l^{2}\right)^{2}-4\left(2-3 r_{+}^{2} / l^{2}\right)\left(3 r_{+}^{10} / l^{10}-r_{+}^{8} / l^{8}-2 r_{+}^{6} / l^{6}\right)}}{2\left(2-3 r_{+}^{2} / l^{2}\right)} .
$$

The thermodynamical stable regions are plotted in Fig. 9. The region enclosed by the two curves starting from $q=0$ and $r_{+} / l=1$ are thermodynamic unstable with a negative heat capacity for a fixed charge $C_{\tilde{q}}$. The another thermodynamic unstable region is enclosed by the charge axe $q$, the extremal black hole curve and the one starting from $q=0$ and $r_{+}=0$ and going to infinity at $r_{+} / l=\sqrt{2 / 3}$. In other regions the hyperbolic black hole is locally thermodynamically stable with a positive heat capacity $C_{\tilde{q}}$.

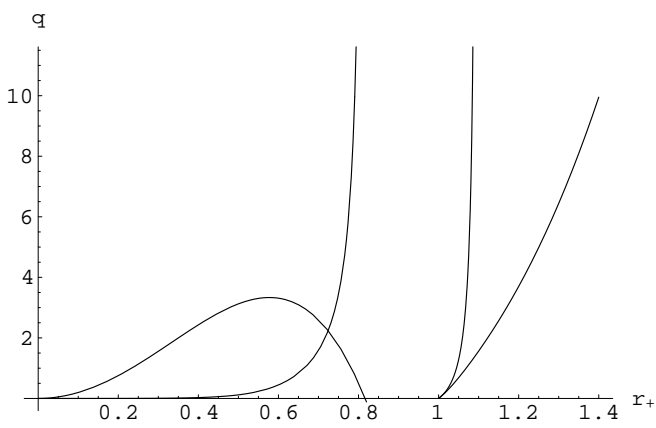

FIG. 9: The curves denote the charge $q / l^{4}$ versus the horizon radius $r_{+} / l$. In the region enclosed by the two curves starting at $q=0$ and $r_{+} / l=1$ the black hole is thermodynamically unstable with a negative heat capacity $C_{\tilde{q}}$. The black hole is also locally unstable in the region which is enclosed by the charge axe $q$, the extremal black hole curve and the one starting at $q=0$ and $r_{+}=0$ and going to infinity at $r_{+} / l=\sqrt{2 / 3}$.

\section{CONCLUSIONS AND DISCUSSIONS}

We discussed the thermodynamics, global and local stability of hyperbolic charged black holes in AdS spaces. These black holes include hyperbolic RN black holes in arbitrary dimensions, hyperbolic charged black holes in $\mathrm{D}=5$, 4 and 7 dimensional gauged supergravities. In particular, we emphasized how to choose appropriate parameters to parameterize the solution in the isotropic coordinates so that the chosen parameters can cover the whole phase space. 
We found that the entropy of the hyperbolic RN black holes can be expressed by a Cardy-Verlinde-like formula, but the Casimir energy is negative, which generalized the discussion for the neutral black holes to the charged case. It is easy to check that similar formula holds for the hyperbolic black holes in $\mathrm{D}=5,4$ and 7 dimensional gauged supergravities.

For the hyperbolic RN black holes in arbitrary dimensions, we found that the Gibbs free energy is always negative and heat capacity with a fixed gauge potential is always positive. This shows that in the grand canonical ensemble, the black hole is stable not only globally but also locally; the Hawking-Page phase transition will not happen and black hole phase is dominant in the dual CFTs. In the canonical ensemble, the heat capacity with a given electric charge is still positive, indicating the local stability of the black hole, but the Helmholtz free energy can change its sign. This implies that a Hawking-Page phase transition will occur in the canonical ensemble.

For the hyperbolic black holes in $\mathrm{D}=5,4$ and 7 dimensional gauged supergravities, the negative definiteness of Gibbs free energy still persists, but the heat capacity with a given gauge potential could change its sign. This implies that in the grand canonical ensemble, the hyperbolic black holes are globally stable, but not locally. The black hole phase is globally preferred and dominant in the dual CFTs, but the black holes cannot always be thermal equilibrium with an infinite thermal bath. In the canonical ensemble, there is always a region where the heat capacity with a given electric charge is negative, indicating the local instability of black holes. The Helmholtz free energy for a single charge black holes can change its sign, except for the case of $D=4$. This implies that a Hawking-page phase transition will appear as the case of hyperbolic RN black holes. We noted that the Helmholtz free energy is always negative for a single charged black hole in $\mathrm{D}=4$ gauged supergravity. To understand this situation, it is helpful to recall the case of rotating M2-branes [13, 14, 15], there in the case of a single rotation parameter, the free energy is also always negative, but it will change its sign for multiple rotation parameters. According to the relationship between the black hole solutions in $\mathrm{D}=4$ gauged supergravity and the rotating M2-branes [6, 14]) (also see [19]), we expect that the Helmholtz free energy will also change its sign for multiple charged hyperbolic black holes in $\mathrm{D}=4$ gauged supergravity, which in fact is already predicted by (4.9).

We point out here that when analyzing the stability and phase structure of a single charged black holes in $\mathrm{D}=5,4$ and 7 gauged supergravities, we restricted ourselves to the case $q>0$. As we discussed in the text, for the hyperbolic black holes, it is also possible to have $q<0$, and in this case the solution still has a black hole structure. Therefore it would be of interest to extend the discussion to the case of $q<0$, and also to the case of multiple charges. Further, the implication in the AdS/CFT correspondence is worth investigating [23].

When discussing the global stability of AdS black holes, one has to calculate the Euclidean action associated with the black hole. Due to the infinite volume, the Hilbert-Einstein action for the black hole configuration is divergent. To get a finite Euclidean action, usually one can use the so-called background subtraction approach 20], or the surface counterterm approach 24]. In the background subtraction approach, one has to choose an appropriate background: for asymptotically flat spaces, the Minkowski space is a suitable one [25], while for asymptotically AdS spaces, the AdS space is a suitable choice. But in some cases, for example, for the Taub-NUT and Taub-Bolt spaces, an appropriate vacuum background is difficult to choose. For those cases, the surface counterterm approach works very well [24]. In this paper, we have used the background substraction approach [20]. The chosen background is the AdS vacuum solution with vanishing mass parameter and charge parameter. When calculating the gravitational mass of the hyperbolic black hole, we used the formula (3.12), which is derived from the Euclidean action of black holes by subtracting the contribution of chosen vacuum background [20]. Through such defined mass, the resulting free energies associated with black holes have the relation to the Euclidean action of black holes as $\mathcal{G}=T \mathcal{I}_{g}$ and $\mathcal{F}=T \mathcal{I}_{c}$ in the grand canonical ensemble and canonical ensemble [17], respectively. Here the Euclidean actions $\mathcal{I}_{g}$ and $\mathcal{I}_{c}$ are understood as the differences between the action of black holes and the one for the vacuum background (see for example [17, 25]). Therefore, the Euclidean action and free energy have the same signs and then the global stability of AdS black holes can be inferred from the behavior of free energy in the grand canonical ensemble and canonical ensemble. For the equivalence, one may further refer to [4, 11, 14]. For the hyperbolic AdS black holes, the choice of the vacuum background is not unique as we pointed out before. One of the choices is to take the extremal black hole as the background (see for example [4]). Another natural choice is just to take the AdS background without any excitation, namely black hole solutions with vanishing mass parameter (we chose this background). The surface counterterm approach reveals that both the vacuum backgrounds might be suitable [26]. Anyway, it is an interest issue to further study the dependence of global stability on the choice of vacuum background. In particular, it is of great importance to investigate whether or not there is a mechanism to uniquely determine a physical vacuum background via the AdS/CFT correspondence.

Finally we stress that when the heat capacities are negative, both the grand canonical ensemble and canonical ensemble are not well-defined. In that case, one way to remedy this difficulty is to put the AdS black hole in a finite cavity as the case of a Schwarzschild black hole in a cavity (see the paper by York in [25]). In this paper, we are also interested in thermodynamics and stability of dual CFTs which reside on the boundary of AdS space, so the cavity is put on the boundary of AdS space. For large AdS Schwarzschild black holes [9, 11], the heat capacity 
is positive. Therefore it is well-defined to discuss thermodynamics and stability of the black hole in the canonical ensemble. For small AdS Schwarzschild black holes, the heat capacity is negative. This of course implies that one cannot use the canonical ensemble to study the small AdS Schwarzschild black hole. Instead it is understood that this small black hole with negative heat capacity cannot be in thermal equilibrium with an infinite heat bath, as the case of Schwarzschild black hole. In the AdS/CFT correspondence, on the side of dual CFTs, the phase of small black hole should be replaced by a thermal AdS background, which has a positive heat capacity [9, 11]. In our case, the situation is similar to the case of AdS Schwarzschild black hole. The hyperbolic charged black holes with heat capacity cannot be in thermal equilibrium with an infinite heat bath surrounding the black hole. On the side of dual CFTs, the corresponding phase is not controlled by these black holes with negative heat capacity, but by other gravity configurations, for example a thermal AdS background with a fixed gauged potential. No doubt it is of interest to study thermodynamics and stability of these hyperbolic charged black holes in a finite cavity as the cases of charged AdS black holes with positive constant curvature horizon [3, 27] and with zero curvature horizon [28]. In

particular, one should be quite interested in whether or not the thermodynamics of AdS black hole in a cavity has a dual description on the side of dual CFTs.

\section{Acknowledgments}

RGC would like to thank J.X. Lu and W. Sabra for helpful discussions at the initial stage of this work. He also would like to express his gratitude to the Physics Department, Baylor University for its hospitality. This work was supported by Baylor University, a grant from Chinese Academy of Sciences, a grant from NSFC, China (No. 10325525), and a grant from the Ministry of Science and Technology of China (No. TG1999075401).

[1] J. L. Friedman, K. Schleich and D. M. Witt, Phys. Rev. Lett. 71, 1486 (1993) [Erratum-ibid. 75, 1872 (1995)] arXiv:gr-qc/9305017; T. Jacobson and S. Venkataramani, Class. Quant. Grav. 12, 1055 (1995) arXiv:gr-qc/9410023.

[2] J. P. S. Lemos, Phys. Lett. B 353, 46 (1995) arXiv:gr-qc/9404041; J. P. Lemos, Class. Quant. Grav. 12, 1081 (1995) gr-qc/9407024; J. P. S. Lemos and V. T. Zanchin, Phys. Rev. D 54, 3840 (1996) arXiv:hep-th/9511188. C. G. Huang and C. B. Liang, Phys. Lett. A 201 (1995) 27; R. G. Cai and Y. Z. Zhang, Phys. Rev. D 54, 4891 (1996) arXiv:gr-qc/9609065; R. G. Cai, J. Y. Ji and K. S. Soh, Phys. Rev. D 57, 6547 (1998) arXiv:gr-qc/9708063. R. G. Cai, Nucl. Phys. B 524, 639 (1998) arXiv:gr-qc/9801098; S. Aminneborg, I. Bengtsson, S. Holst and P. Peldan, Class. Quant. Grav. 13, 2707 (1996) gr-qc/9604005. R. B. Mann, Class. Quant. Grav. 14, L109 (1997) gr-qc/9607071; R. B. Mann, Nucl. Phys. B 516, 357 (1998) hep-th/9705223. L. Vanzo, Phys. Rev. D 56, 6475 (1997) gr-qc/9705004. D. Klemm, Class. Quant. Grav. 15, 3195 (1998) gr-qc/9808051; D. Klemm, V. Moretti and L. Vanzo, Phys. Rev. D 57, 6127 (1998) [Erratumibid. D 60, 109902 (1998)] gr-qc/9710123; M. Banados, A. Gomberoff and C. Martinez, Class. Quant. Grav. 15, 3575 (1998) hep-th/9805087. M. F. A. da Silva, A. Wang, F. M. Paiva and N. O. Santos, Phys. Rev. D 61, 044003 (2000) arXiv:gr-qc/9911013 ; R. G. Cai, Phys. Lett. B 572, 75 (2003) arXiv:hep-th/0306140; R. Aros, R. Troncoso and J. Zanelli, Phys. Rev. D 63, 084015 (2001) hep-th/0011097. R. G. Cai, Phys. Rev. D 65, 084014 (2002) arXiv:hep-th/0109133. R. G. Cai, Phys. Lett. B 582, 237 (2004) arXiv:hep-th/0311240; W. L. Smith and R. B. Mann, Phys. Rev. D 56, 4942 (1997) gr-qc/9703007; Y. Wu, M. F. A. da Silva, N. O. Santos and A. Wang, Phys. Rev. D 68, 084012 (2003) arXiv:gr-qc/0309002 ; S. Nojiri and S. D. Odintsov, Phys. Lett. B 521, 87 (2001) [Erratum-ibid. B 542, 301 (2002)] arXiv:hep-th/0109122; M. Cvetic, S. Nojiri and S. D. Odintsov, Nucl. Phys. B 628, 295 (2002) arXiv:hep-th/0112045. S. Nojiri and S. D. Odintsov, Phys. Rev. D 66, 044012 (2002) arXiv:hep-th/0204112. Y. M. Cho and I. P. Neupane, Phys. Rev. D 66, 024044 (2002) arXiv:hep-th/0202140. I. P. Neupane, Phys. Rev. D 67, 061501 (2003) arXiv:hep-th/0212092. I. P. Neupane, arXiv:hep-th/0302132 and references therein.

[3] D. R. Brill, J. Louko and P. Peldan, Phys. Rev. D 56, 3600 (1997) arXiv:gr-qc/9705012;

[4] D. Birmingham, Class. Quant. Grav. 16, 1197 (1999) hep-th/9808032.

[5] R. Cai and K. Soh, Phys. Rev. D 59, 044013 (1999) gr-qc/9808067;

[6] A. Chamblin, R. Emparan, C. V. Johnson and R. C. Myers, Phys. Rev. D 60, 064018 (1999) arXiv:hep-th/9902170;

[7] M. M. Caldarelli and D. Klemm, Nucl. Phys. B 545, 434 (1999) arXiv:hep-th/9808097.

[8] R. G. Cai, Phys. Rev. D 63, 124018 (2001) arXiv:hep-th/0102113.

[9] S. W. Hawking and D. N. Page, Commun. Math. Phys. 87, 577 (1983).

[10] J. Maldacena, Adv. Theor. Math. Phys. 2, 231 (1998) [Int. J. Theor. Phys. 38, 1113 (1998)] hep-th/9711200; S. S. Gubser, I. R. Klebanov and A. M. Polyakov, Phys. Lett. B 428, 105 (1998) hep-th/9802109; E. Witten, Adv. Theor. Math. Phys. 2, 253 (1998) hep-th/9802150.

[11] E. Witten, Adv. Theor. Math. Phys. 2, 505 (1998) arXiv:hep-th/9803131.

[12] S. S. Gubser, Nucl. Phys. B 551, 667 (1999) arXiv:hep-th/9810225.

[13] R. G. Cai and K. S. Soh, Mod. Phys. Lett. A 14, 1895 (1999) arXiv:hep-th/9812121.

[14] M. Cvetic and S. S. Gubser, JHEP 9904, 024 (1999) arXiv:hep-th/9902195. 
[15] M. Cvetic and S. S. Gubser, JHEP 9907, 010 (1999) arXiv:hep-th/9903132.

[16] T. Harmark and N. A. Obers, JHEP 0001, 008 (2000) arXiv:hep-th/9910036.

[17] H. W. Braden, J. D. Brown, B. F. Whiting and J. W. . York, Phys. Rev. D 42, 3376 (1990).

[18] K. Behrndt, M. Cvetic and W. A. Sabra, Nucl. Phys. B 553, 317 (1999) arXiv:hep-th/9810227.

[19] M. Cvetic et al., Nucl. Phys. B 558, 96 (1999) arXiv:hep-th/9903214; M. J. Duff, arXiv:hep-th/9912164

[20] J. D. Brown and J. W. . York, Phys. Rev. D 47, 1407 (1993); S. W. Hawking and G. T. Horowitz, Class. Quant. Grav. 13, 1487 (1996) arXiv:gr-qc/9501014]; J. D. Brown, J. Creighton and R. B. Mann, Phys. Rev. D 50, 6394 (1994) arXiv:gr-qc/9405007.

[21] M. J. Duff and J. T. Liu, Nucl. Phys. B 554, 237 (1999) arXiv:hep-th/9901149.

[22] W. A. Sabra, Phys. Lett. B 458, 36 (1999) arXiv:hep-th/9903143.

[23] B. McInnes, Nucl. Phys. B 660, 373 (2003) arXiv:hep-th/0205103.

[24] V. Balasubramanian and P. Kraus, Commun. Math. Phys. 208, 413 (1999) arXiv:hep-th/9902121; R. Emparan, C. V. Johnson and R. C. Myers, Phys. Rev. D 60, 104001 (1999) arXiv:hep-th/9903238; P. Kraus, F. Larsen and R. Siebelink, Nucl. Phys. B 563, 259 (1999) arXiv:hep-th/9906127.

[25] G. W. Gibbons and S. W. Hawking, Phys. Rev. D 15, 2752 (1977); J. W. . York, Phys. Rev. D 33, 2092 (1986).

[26] R. Emparan, JHEP 9906, 036 (1999) arXiv:hep-th/9906040.

[27] C. S. Peca and J. P. S. Lemos, Phys. Rev. D 59, 124007 (1999) arXiv:gr-qc/9805004.

[28] C. S. Peca and J. P. S. Lemos, J. Math. Phys. 41, 4783 (2000) arXiv:gr-qc/9809029. 